\title{
EchoGéo
}

$57 \mid 2021$

Les enjeux de l'alimentation en eau potable des villes

\section{La gestion communautaire de l'eau dans les páramos de Bogota (Colombie)}

Le cas du réseau Piedra Parada y Cerrito blanco

\section{Angela Osorio}

\section{OpenEdition}

Journals

Édition électronique

URL : https://journals.openedition.org/echogeo/22369

DOI : $10.4000 /$ echogeo.22369

ISSN : 1963-1197

Éditeur

Pôle de recherche pour l'organisation et la diffusion de l'information géographique (CNRS UMR 8586)

Référence électronique

Angela Osorio, « La gestion communautaire de l'eau dans les páramos de Bogota (Colombie) », EchoGéo [En ligne], 57 | 2021, mis en ligne le 15 octobre 2021, consulté le 22 novembre 2021. URL : http://journals.openedition.org/echogeo/22369; DOI : https://doi.org/10.4000/echogeo.22369

Ce document a été généré automatiquement le 22 novembre 2021.

EchoGéo est mis à disposition selon les termes de la licence Creative Commons Attribution - Pas d'Utilisation Commerciale - Pas de Modification 4.0 International (CC BY-NC-ND) 


\title{
La gestion communautaire de l'eau dans les páramos de Bogota (Colombie)
}

\author{
Le cas du réseau Piedra Parada y Cerrito blanco
}

Angela Osorio

Une première version de ce texte a été révisée grâce aux précieux conseils de A. Poupard, I. Mestre, S. Houelleu et les suggestions de deux évaluateurs/rices. Je tiens à les remercier.

\section{Introduction}

1 La planète Terre a été profondément modifiée par les activités anthropiques, entraînant, entre autres, la perte de la biodiversité et le déclin des services écosystémiques (Theobald et al., 2020). Dans ce contexte, la création et la gestion des aires protégées ont été l'une des principales stratégies pour faire face aux conséquences importantes de ces modifications depuis le XIX ${ }^{\mathrm{e}}$ siècle à toutes les échelles (Depraz, 2008). La Colombie ne fait pas exception à la tendance mondiale dans ce domaine. Cette situation est illustrée par le processus de délimitation et de protection des páramos, écosystème caractéristique des Andes septentrionales qui fournissent de multiples services écosystémiques (ex: l'approvisionnement et la régulation de l'eau, la conservation de la biodiversité et le stockage du carbone) (Quiroz et al., 2021 ; Mendez, 2019).

2 En Colombie, la loi 99 de 1993 a permis la création du Ministère de l'Environnement, a permis d'organiser le système environnemental national et a imposé la protection des páramos et des sources d'eau. Depuis lors, un nombre important de documents législatifs et de lignes directrices cherchant à encadrer ce processus ont vu le jour (Buitrago, 2016). Cependant, ces documents et lignes directrices ont négligé les liens dérivés de la présence historique des communautés dans ces zones, ont sous-estimé leur rôle dans le façonnement des paysages des hautes montagnes colombiennes et ont rendu responsables les communautés locales de la détérioration des écosystèmes, ce 
qui a généré des tensions entre l'État et les communautés qui habitent les páramos (Mendez, 2019).

3 Depuis quelques décennies, l'intérêt scientifique pour les páramos colombiens s'est considérablement accru notamment en ce qui concerne des aspects comme leur biodiversité (Gil-Leguizamon, et al., 2020 ; Hopfe et al., 2020 ; Olaya-Angarita et al., 2019), leur hétérogénéité spatiale (Duarte-Ballesteros et al., 2021), les impacts du changement climatique sur les écosystèmes (Cresso et al., 2020), leur pollution (Alonso et al., 2020 ; Gonzalez-Martinez et al., 2019) et les caractéristiques de leurs sols (Gutierrez et al, 2020 ; Alvarez-Yela et al., 2017). À cet intérêt scientifique croissant s'ajoutent des mobilisations sociales qui rejettent les projets liés à l'extractivisme des multinationales dans les régions d'influence des páramos et qui plaident pour la protection de ces écosystèmes, comme dans le cas du páramo de Santurbán, qui a été l'un des plus médiatisés et avec la plus grande participation citoyenne de ces dernières décennies (Acevedo-Tarazona et Correa-Lugos, 2019).

4 Cependant, les études scientifiques sur la dimension sociale des páramos colombiens sont beaucoup moins nombreuses. Parmi les rares études, il convient d'en mentionner deux : les travaux de Muñoz-Guerrero (2017) et ceux de Acevedo-Tarazona et CorreaLugos (2019). Muñoz-Guerrero (2017) présente une analyse descriptive des principaux facteurs socio-économiques qui ont été déterminants dans les transformations des paysages du páramo de Paja Blanca en Nariño (Colombie) au cours de la période 1984-2013, tandis que Acevedo-Tarazona et Correa-Lugos (2019) se concentrent sur les actions collectives menées dans le département de Santander (Colombie), en réponse à la question du páramo de Santurbán et à l'intérêt des multinationales pour développer des projets miniers à grande échelle.

5 Concernant plus particulièrement la délimitation des páramos, quelques publications affirment qu'ils doivent être considérés comme des systèmes socio-écologiques et que les communautés qui les habitent doivent être prises en compte dans le processus de délimitation et de gestion de ces territoires (Mendez et al., 2019; Zapata, 2020). La reconnaissance des communautés habitant les páramos a encore plus d'importance dans un contexte de post signature de l'accord de paix entre les autorités et la guérilla des Farc qui date de 2016. En effet, dans ce contexte, le rôle des páramos comme couloir stratégique dans les conflits armés est devenu évident, ainsi que les intérêts miniers dans les páramos qui peuvent générer une dépossession des communautés, surtout si elles ne sont pas reconnues (Buitrago, 2016).

6 La demande de prise en compte des communautés locales est principalement basée sur le fait qu'il existe une demande sociale et que les communautés peuvent jouer un rôle dans la protection de la nature. Bien que certaines études scientifiques aient démontré l'existence d'une relation entre la gestion de l'eau et la protection des páramos dans certains départements du pays comme Santander et Norte de Santander (Soto-Vallejo, 2020) elles n'ont pas été réalisées, à notre connaissance, dans les páramos de Bogota, capitale de la Colombie où les páramos assurent des services écosystémiques essentiels tel que l'approvisionnement en eau. Il s'agit d'une véritable lacune scientifique, d'autant plus que cette zone abrite ce qui est considéré comme le plus grand páramo du monde, avec une superficie de 266750 ha (Morales et al., 2007 ; Villay-Quiñones, 2018). Remédier à cette absence d'étude scientifique serait essentiel afin d'établir une protection des páramos par une approche intégrative de la nature dans laquelle les 
communautés locales occupent un rôle important de protection de l'environnement (Depraz, 2008 ; Therville, 2013).

7 Dans cet article, l'objectif est de décrire la gestion des «systèmes alternatifs d'approvisionnement en eau », pour utiliser les termes de Coutard (2010). Ce travail a été développé dans le cadre d'un stage de recherche pour l'obtention du diplôme de Master en Géographie et Aménagement du territoire à l'Université Jean Moulin Lyon 3, pour l'année universitaire 2015-2016. Ce stage a été effectué au sein de l'Observatoire de l'eau et de la ville de l'ONG ENDA-Colombie. Les techniques de recueil des données ont été les suivantes :

- une analyse documentaire (travaux scientifiques, documents officiels, documents fournis par l'ONG ENDA-Colombie et par les conseils d'administration des réseaux d'adduction d'eau) ;

- l'observation directe de terrain (lors des visites des réseaux d'adduction d'eau et la participation à différentes réunions organisées par le réseau territorial des réseaux communautaires de Bogota (RETACO) et l'Empresa de Acueducto y Alcantarillado de Bogota, entité publique qui administre les systèmes publics d'approvisionnement et d'assainissement de la ville de Bogota);

- des entretiens semi-directifs auprès de différents acteurs (personnel de l'ONG ENDAColombie, administrateurs et usagers des réseaux d'adduction d'eau);

- des ateliers de cartographie participative, un avec les gestionnaires et l'autre avec des usagers du réseau Piedra Parada y Cerrito blanco.

8 Je commencerai par présenter les réseaux d'approvisionnement alternatifs existants à Bogota. Ensuite, je mettrai l'accent sur l'étude de cas d'un système alternatif d'approvisionnement en eau situé dans un páramo de Bogota. La description du réseau se fera en prenant comme cadre d'analyse la théorie des biens communs d'Elinor Ostrom (1990).

\section{Les réseaux d'adduction d'eau alternatifs à la gestion publique centralisée à Bogota}

Selon le droit colombien, la mise à disposition des services publics à domicile est une responsabilité de l'État mais avec la loi 142 de 1994, la possibilité aux tiers de participer à l'approvisionnement est apparue dans la législation. Cette ouverture est liée à au moins deux facteurs: (i) les processus de privatisation des services de l'eau et (ii) la couverture insuffisante des services d'eau et d'assainissement dans tout le pays, qui persiste même après l'entrée en vigueur de cette loi (Montoya-Domínguez et RojasRobles, 2019).

La capitale du pays, Bogota, ne fait pas exception à la couverture insuffisante des services d'eau et d'assainissement, notamment en raison de l'importante demande en eau générée par les plus de 8 millions d'habitants. Dans cette ville, il existe un fournisseur public principal qui alimente les quartiers urbains et certaines zones rurales, mais l'augmentation de la demande a entraîné un accroissement du nombre de communautés qui fournissent de l'eau par leurs propres moyens (Llano-Arias, 2015) notamment dans les zones rurales qui correspondent à $75 \%$ de la surface totale de Bogota, soit 122000 des 163000 hectares (Secretaria Distrital del ambiente, 2021). 
11 Dans ce contexte, un recensement des systèmes d'approvisionnement alternatifs à la gestion publique centralisée était essentiel pour avoir un panorama de la gestion de l'eau non centralisée à Bogota. Pour cela un géo-référencement de l'infrastructure des réseaux d'approvisionnement d'eau alternatifs a été réalisé. Ce géo-référencement a permis d'identifier 75 réseaux dans 10 des 20 arrondissements de la capitale ${ }^{1}$. Chacun de ces réseaux a été caractérisé en fonction de la localisation géographique, des activités économiques associées, du type d'habitat (pour les réseaux alimentant les ménages), du nombre d'usagers, des risques pouvant impacter la qualité de l'eau et la manière dont les usagers revendiquent l'existence du réseau. Ces informations ont révélé l'existence de différences majeures entre les réseaux qui méritent d'être mises en évidence au moyen d'une typologie. Une typologie basée sur la théorie de la nouvelle culture de l'eau (Arrojo, 2008) a été proposée, dont le résultat est présenté cidessous :

- Type de réseau I : le réseau approvisionne en eau des communautés paysannes en milieu rural et dans certaines habitations périurbaines. La gestion du réseau est revendiquée par les habitants au titre du droit fondamental à l'eau. 37 réseaux correspondent à ce type.

- Type de réseau II : le réseau approvisionne en eau des quartiers aisés construits dans des zones périurbaines et rurales. La gestion du réseau est revendiquée par les habitants par l'exercice des droits des citoyens, tels que le droit d'association et de participation. Huit réseaux correspondent à ce type.

- Type de réseau III : le réseau approvisionne en eau des zones urbaines et périurbaines pour le développement d'activités économiques et éducatives (par exemple, le réseau fournit de l'eau aux entreprises ou aux écoles pour l'exécution de leur travail). Le réseau fonctionne de manière parallèle aux réseaux d'approvisionnement publics centralisés. Neuf réseaux correspondent à ce type.

- Type de réseau IV : le réseau approvisionne en eau certains bâtiments des institutions publiques. Le réseau fonctionne de manière parallèle aux réseaux d'approvisionnement publics centralisés. Cinq réseaux correspondent à ce type.

12 Selon les critères proposés par l'Institut de recherche Alexandre Von Humboldt (Rivera et Rodríguez, 2011) et conformément aux travaux scientifiques récents (par exemple, Gil-Leguizamon, 2020), l'emplacement de l'infrastructure des réseaux appartenant aux quatre types a été évalué afin de déterminer lesquels d'entre eux se trouvent dans les zones de páramo. En effet, cet institut de recherche considère que la zone d'écotone du páramo bas ou du sous-páramo se situe approximativement à partir de 2800 mètres d'altitude. Aussi pour cette analyse les coordonnées et l'altitude exacte de tous les réseaux réunis ont été utilisés. Cette analyse a révélé que l'infrastructure de 19 réseaux se trouve entre 2800 et 3739 mètres d'altitude.

Sur la base de ces données, un contact a été établi avec le réseau territorial des réseaux communautaires de Bogota (RETACO). RETACO est né en 2009, suite à la campagne référendaire pour l'eau de la même année (Llano-Arias, 2015) qui visait la reconnaissance de l'eau potable comme un droit fondamental, la fourniture d'un minimum vital gratuit, la protection spéciale des écosystèmes essentiels pour le cycle de l'eau, et la gestion publique, étatique et communautaire des services d'eau et d'assainissement. Ce référendum a été rejeté par le gouvernement en place mais a généré, selon les discussions directes avec le président de RETACO, une importante organisation sociale des réseaux d'approvisionnement en eau. Ces derniers ont su 
collectivement répondre aux différents scandales sur la qualité de l'eau fournie, par exemple en 2010 certains réseaux alternatifs ont été accusés de fournir de l'eau contaminée par des bactéries coliformes (El tiempo, 2010) et en 2011 les allégations de contamination étaient liées à la présence de mercure (Aguilar-Torres, 2012). Pour faire face à ces allégations, RETACO a établi des partenariats avec diverses organisations publiques et non gouvernementales pour surveiller et améliorer la qualité de l'eau.

La relation avec RETACO m'a permis d'entrer en contact direct avec les gestionnaires et les utilisateurs de certains des réseaux situés dans les páramos et ainsi pouvoir avoir accès directement au terrain. Grâce à cet accès, j'ai pu entrer en contact avec le réseau Piedra Parada y Cerrito blanco dont les gestionnaires et certains usagers se sont montrés intéressés par ma démarche de recherche. J'ai décidé donc de prendre ce réseau comme cas d'étude pour décrire les systèmes alternatifs d'approvisionnement en eau à Bogota. Le cadre d'analyse retenu pour procéder à cette description est celui de la théorie des biens communs d'Elinor Ostrom (1990). Celle-ci a proposé, en opposition à la théorie conventionnelle, la possibilité de développer une gestion de ressources communes, à travers l'action collective, qui soit compatible avec l'environnement et qui soit durable sur le long terme. E. Ostrom (1990) indique que tous les projets de gestion communautaire n'aboutissent pas à une gestion adéquate des ressources. L'auteure a également montré que la gestion communautaire qui aboutit semble suivre les huit principes suivants : ( $\mathrm{P} 1)$ limites clairement définies ; $(\mathrm{P} 2)$ la cohérence entre les règles d'appropriation et de mise à disposition en conformité avec les conditions locales ; (P3) arrangements de choix collectifs; (P4) supervision; (P5) sanctions graduées; (P6) mécanismes de résolution des conflits; (P7) reconnaissance minimales des droits de l'organisation ; et (P8) lien avec d'autres institutions à plusieurs niveaux.

Ce cadre d'analyse s'est avéré pertinent pour ce type de recherches en Colombie comme l'ont montré Delgado-Serrano et al. (2017) à travers une analyse de la gestion communautaire effectuée par l'Association des usagers de l'eau de Mondomo dans le département du Cauca (Colombie). Dans cet article, les auteurs montrent que les principes de conception d'Ostrom peuvent être présents à un certain moment et qu'il est nécessaire de les évaluer de manière qualitative et évolutive plutôt que sur leur absence ou présence. En effet, des changements dans le cadre politique et juridique peuvent survenir et faire évoluer la gestion communautaire des ressources naturelles comme celle de l'eau. Ces possibles fluctuations devraient être prises en compte dans l'analyse.

\section{Cas d'étude : le réseau Piedra Parada y Cerrito blanco}

Le réseau Piedra Parada y Cerrito blanco se trouve au sud-ouest de la ville de Bogota dans l'arrondissement 19-Ciudad Bolivar (illustration 1) et fournit de l'eau potable aux 225 habitations, c'est-à-dire environ 660 bénéficiaires des secteurs Paso Colorado, Alto Lagunitas et Cerrito blanco. Cet arrondissement se caractérise par sa ruralité $(73,9 \% \mathrm{du}$ total de son territoire est classé comme rural) et par son importance environnementale en raison de son altitude qui varie entre les 2400 et $3700 \mathrm{~m}$ (Secretaría Distrital de Planeación, 2009). 


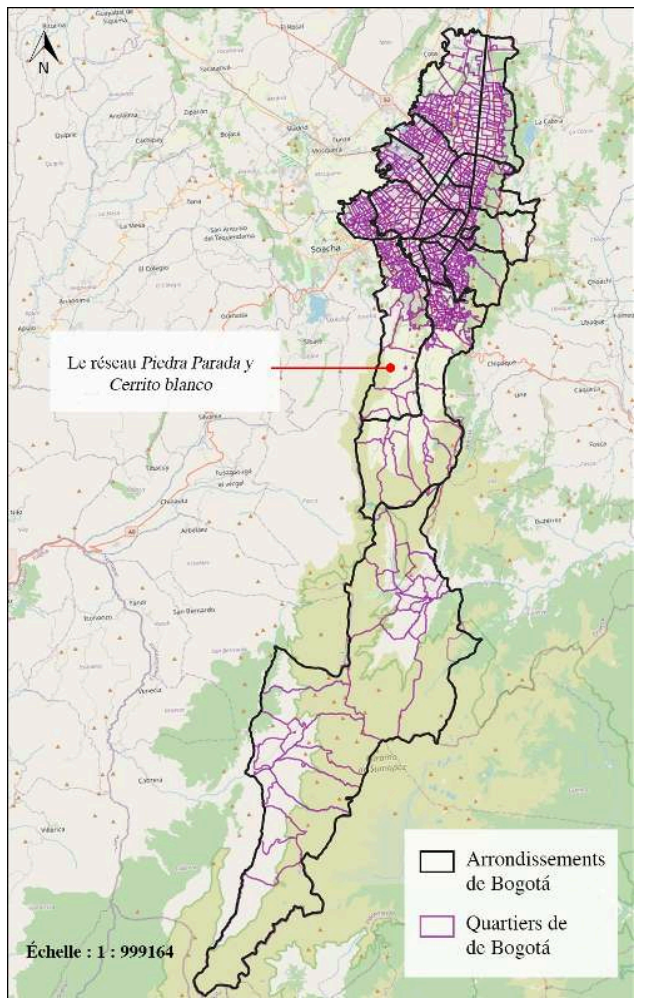

Fond de carte : OpenStreetMap

17 L'arrondissement de Ciudad Bolivar, a été depuis la décennie de 1950, le territoire d'accueil des familles les moins aisées de Bogota et des familles qui échappent de la violence d'autres régions (Londoño-Toro, 2004 ; Gutierrez y Fernandez, 2009). Le réseau Piedra Parada y Cerrito blanco est le résultat d'un processus historique d'actions de la communauté locale pour l'approvisionnement en eau. En effet, dès la seconde moitié du $\mathrm{XX}^{\mathrm{e}}$ siècle la construction massive de logements informels a commencé dans cette zone et il n'existait aucune infrastructure pour amener l'eau des fleuves jusqu'aux habitations.

Chaque jour les femmes, accompagnées des enfants, étaient responsables d'aller jusqu'aux sources pour recueillir l'eau nécessaire à la consommation familiale. Puis, la communauté s'est organisée pour construire de manière collective des tranchées. Celles-ci ont permis à la communauté d'amener l'eau jusqu'aux habitations et de diminuer le temps dédié à la collecte. Cependant, cela n'a pas garanti un accès à l'eau suffisant, ni en quantité, ni en qualité. Pour répondre à ce besoin, la communauté a évolué vers la construction d'une infrastructure plus performante depuis 2009 assurant ainsi la continuité du service, la qualité de l'eau et l'accès à 17 mètres cube par famille (illustration 2). 
Illustration 2 - L'évolution de l'infrastructure du réseau (Bogota, 2016)

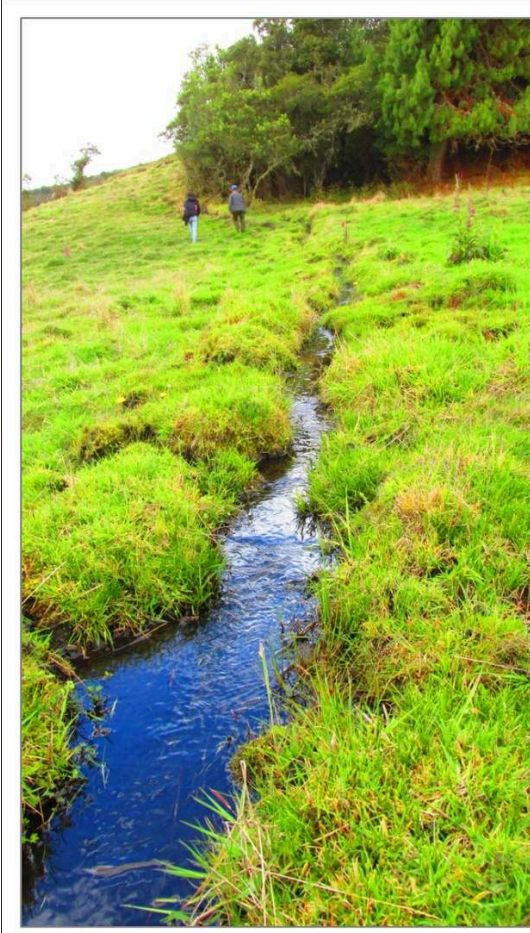

Tranchée faite par la Minga

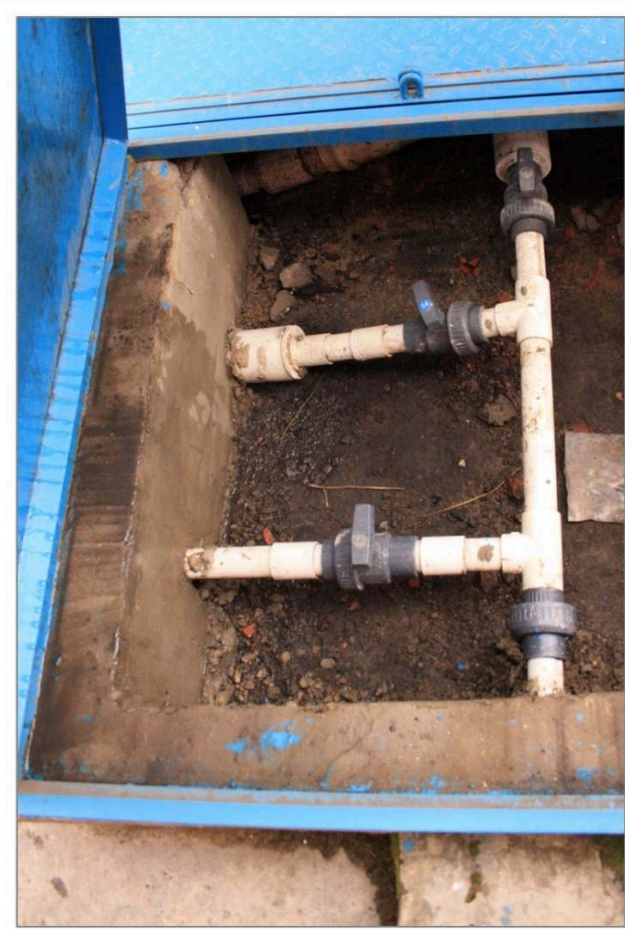

Tubes de distribution actuels

19 Au fil du temps le réseau a intégré des nouveaux instruments et techniques pour l'amélioration du processus de potabilisation de l'eau. Ces améliorations ont été financées par les usagers du réseau et par des donations de l'ONG ENDA-Colombia. En ce qui concerne la formation technique des gestionnaires du réseau, cela a été possible grâce à certains cours de formation dispensés par cette même ONG et l'Empresa de Acueducto y Alcantarillado de Bogota. Un exemple d'amélioration technique est celui des instruments utilisés pour obtenir un goutte-à-goutte du chlore. En effet, au début, ce processus se faisait avec un câble qui servait normalement à l'hydratation du bétail et aujourd'hui on utilise un appareil spécifique qui permet de mesurer exactement la quantité de chlore délivrée (illustration 3). 
Illustration 3 - Instruments utilisés pour le goutte-à-goutte du chlore (Bogota, 2016)

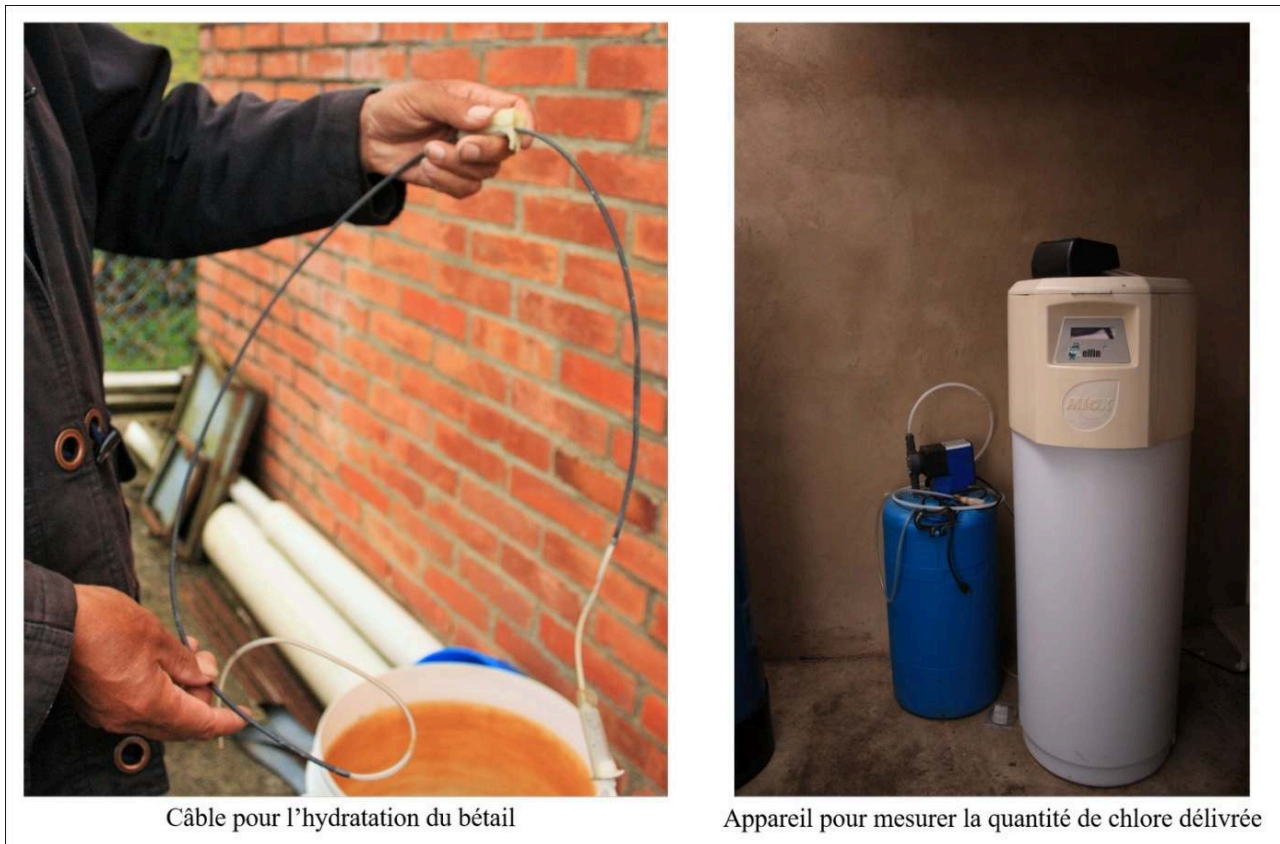

20 Aujourd'hui, les zones de couverture du réseau Piedra Parada et Cerrito blanco sont clairement définies (illustration 4). Cette figure montre une des cartes réalisées par les gestionnaires du réseau dans un des ateliers de cartographie participative. En outre, le réseau dispose d'un enregistrement des 225 usagers et la quantité d'eau que chaque usager peut consommer est déterminée par la législation, ce qui montre que le réseau a des limites clairement définies (P1). Cependant, selon le président du réseau, la quantité d'eau garantie à chaque usager pourrait être remise en cause dans les années à venir, comme l'indique l'extrait d'un entretien réalisé avec lui :

«L'État nous a donné la permission de capturer 0,97 litres par seconde mais cette quantité n'est pas suffisante pour fournir de l'eau à tous les usagers. Alors en 2010 j'ai fait une demande pour augmenter cette quantité mais je n'ai pas encore reçu de réponse. Par ailleurs, l'année dernière chaque usager avait le droit de consommer $20 \mathrm{~m}^{3}$ par mois mais à partir de janvier de cette année le maire a dit qu'on ne pouvait consommer que $17 \mathrm{~m}^{3}$ et que l'année prochaine la consommation va descendre à $11 \mathrm{~m}^{3}$... Il a fait une réduction importante" (Président du réseau, Bogota, 2016). 
Illustration 4 - L'infrastructure et la zone de couverture du réseau Piedra Parada et Cerrito Blanco, résultat de l'atelier de cartographie participative

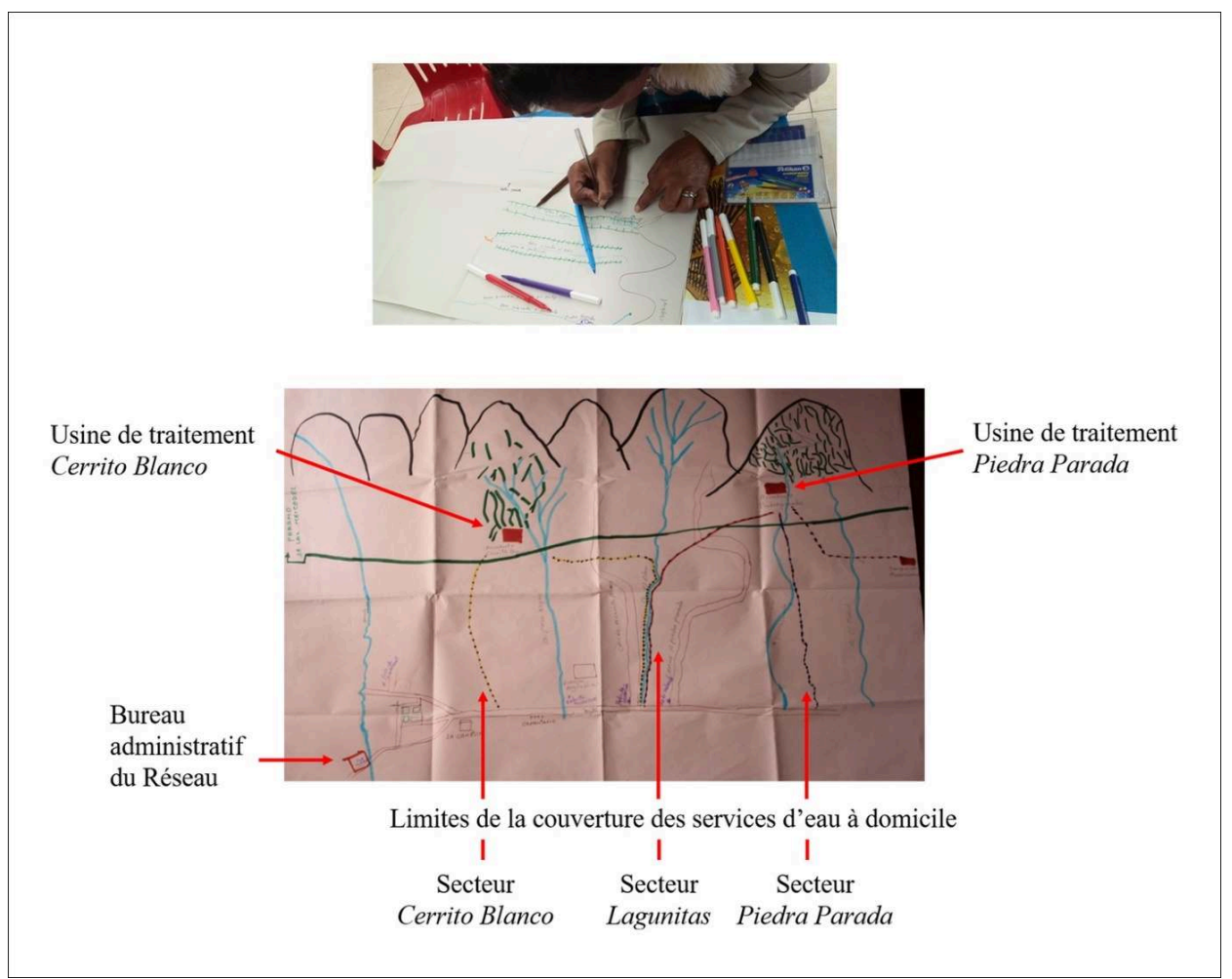

21 Concernant les tarifs pour la prestation du service, ils sont définis en fonction du nombre d'utilisateurs du réseau (P2) comme indiqué par le président du réseau :

«Plus il y a d'utilisateurs, plus les coûts sont bas. Depuis la fusion des deux réseaux (Piedra Parada et Cerrito Blanco), nous avons pu réduire les coûts. Maintenant, tous les utilisateurs paient la même chose » (Président du réseau, Bogota, 2016).

Un autre aspect qui montre la conformité du réseau avec les conditions locales (P2) est le lieu choisi pour l'installation de l'infrastructure et le captage de l'eau. En fait, ces choix ont été faits sur la base des connaissances de la communauté sur le milieu environnant et des accords possibles avec les propriétaires fonciers. L'infrastructure du réseau Piedra Parada et Cerrito Blanco se trouve à proximité de cinq ruisseaux dont deux sont utilisés pour la capture d'eau. De plus, leur infrastructure (le point de captage, le réservoir de stockage, l'usine de traitement des eaux et une partie des tuyaux de conduite) est située sur le Páramo de las Mercedes (illustration 5). 
Illustration 5 - Structure écologique principale qui entoure l'infrastructure du réseau Piedra Parada y Cerrito Blanco

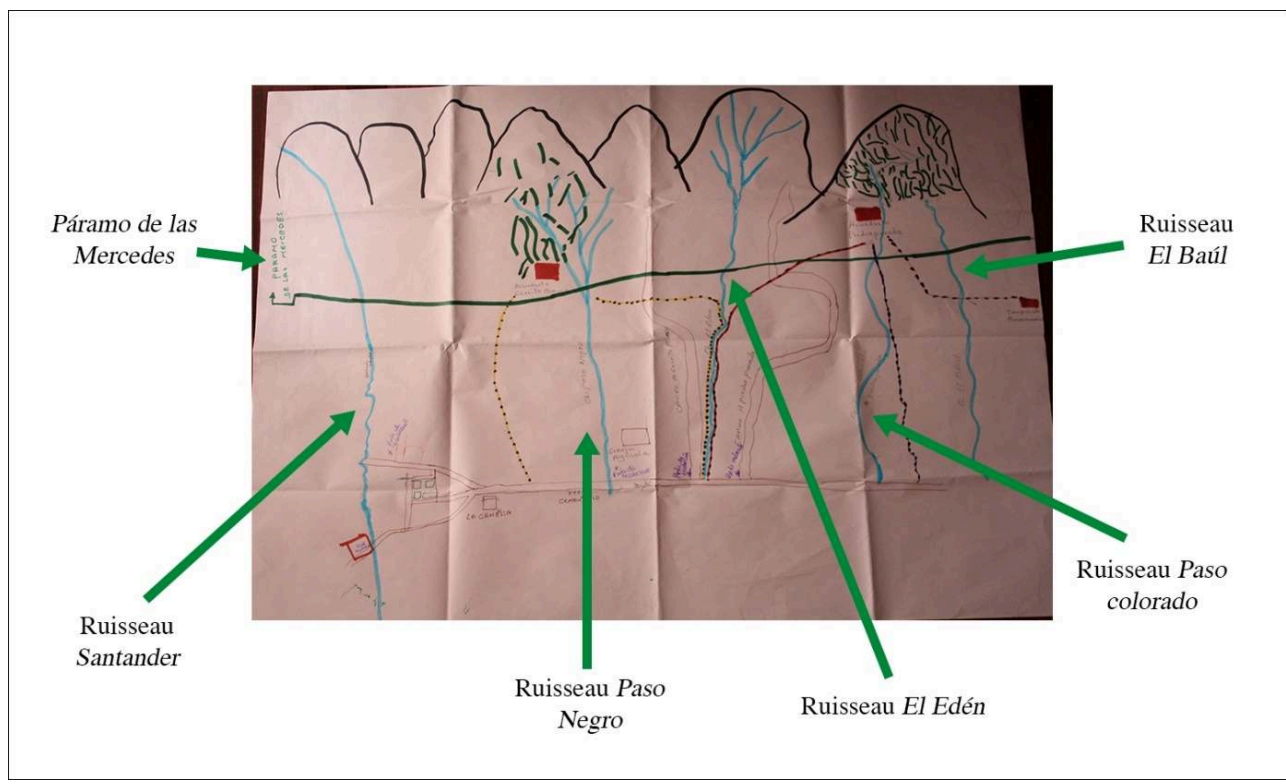

Afin d'assurer la continuité du service en termes de quantité et de qualité, les gestionnaires et les usagers ont suscité un intérêt particulier pour le maintien de la protection de l'environnement autour de l'infrastructure du réseau car c'est grâce à celui-ci qu'ils disposent de la ressource hydrique. Pour y parvenir deux types d'actions ont été identifiés :

(i) la construction des barrières pour limiter l'accès aux ruisseaux, avec l'implatation de haies végétales, pour éviter que le bétail pollue les cours d'eau (illustration 6);

(ii) des projets de reboisement du Páramo où, à cause des actions anthropiques, l'écosystème était déboisé. Pour ces reboisements, le réseau a mis en place des partenariats avec le Jardin botanique de Bogota afin d'obtenir des arbres natifs, qui ont ensuite été plantés par la communauté locale (illustration 7). Les deux extraits d'entretien suivant font référence aux reboisements :

«Il y a eu plusieurs projets de reboisement depuis 2004. Le dernier reboisement date de l'année dernière. Nous avons fait la gestion avec le Jardin Botanique et les unités municipales d'assistance technique pour l'agriculture et l'élevage pour nous aider. Ainsi, nous semons des arbres seulement natifs » (Président du réseau, Bogota, 2016).

«Avec le réseau on a semé des arbres en haut du Páramo. Je connais bien ce travail parce que mon mari a participé aux semis et moi, je faisais les déjeuners pour les personnes qui travaillaient là-bas » (Usagère du réseau, Bogota, 2016). 
Illustration 6 - Les actions de protection de l'écosystème : les haies vives

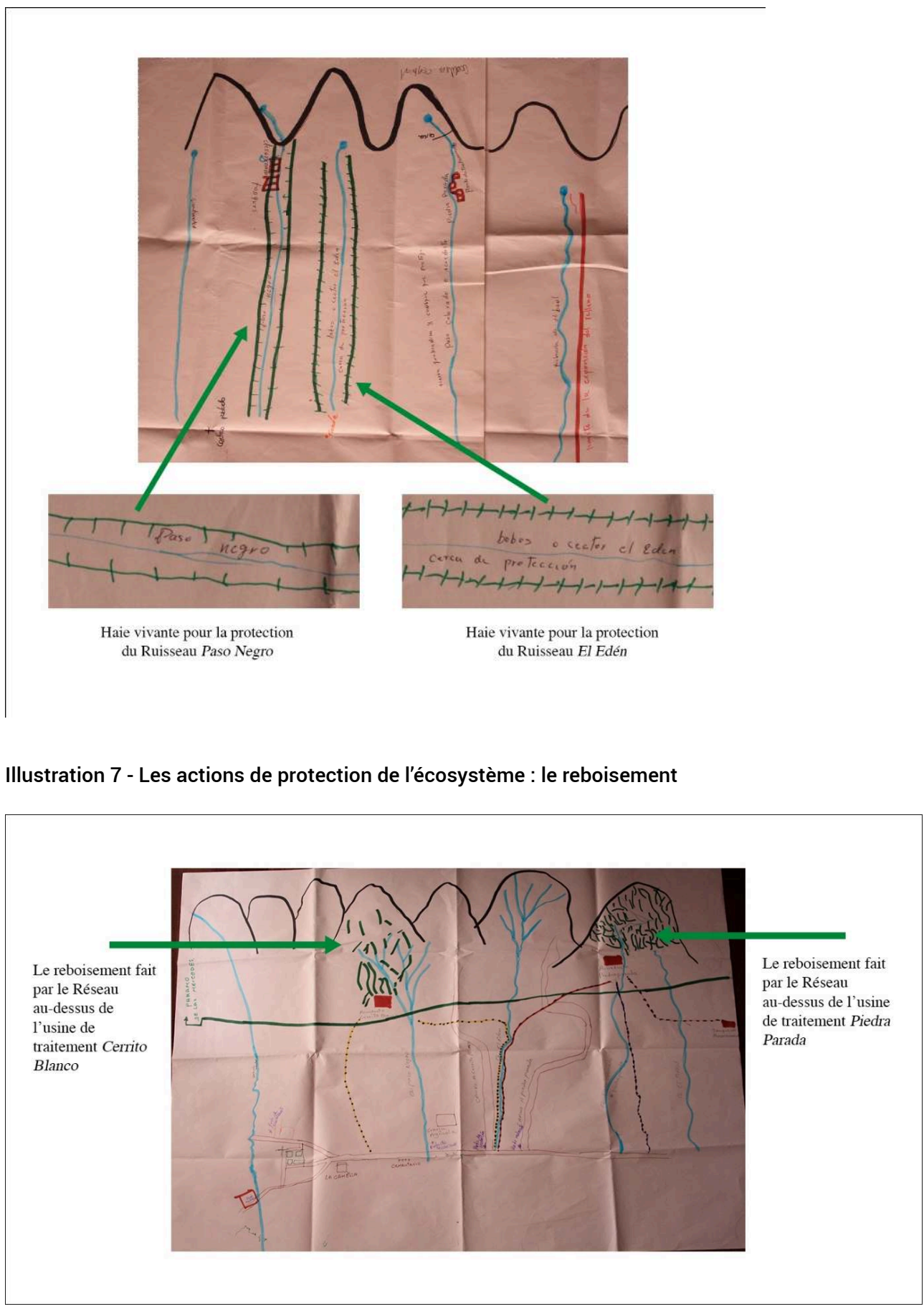

Ces actions montrent qu'à travers le réseau Piedra Parada et Cerrito Blanco des moyens de protection et de restauration écologique ont été mis en œuvre. Ces actions visent la pérennité des services écosystémiques tels que la régulation du cycle hydrologique. Les résultats de cette protection environnementale ont permis de conserver, autour de l'infrastructure du réseau, une biodiversité caractéristique du páramo (illustrations 8 et 9). 
Illustration 8 - Source d'eau du réseau Piedra Parada et Cerrito blanco (Bogota, 2016)

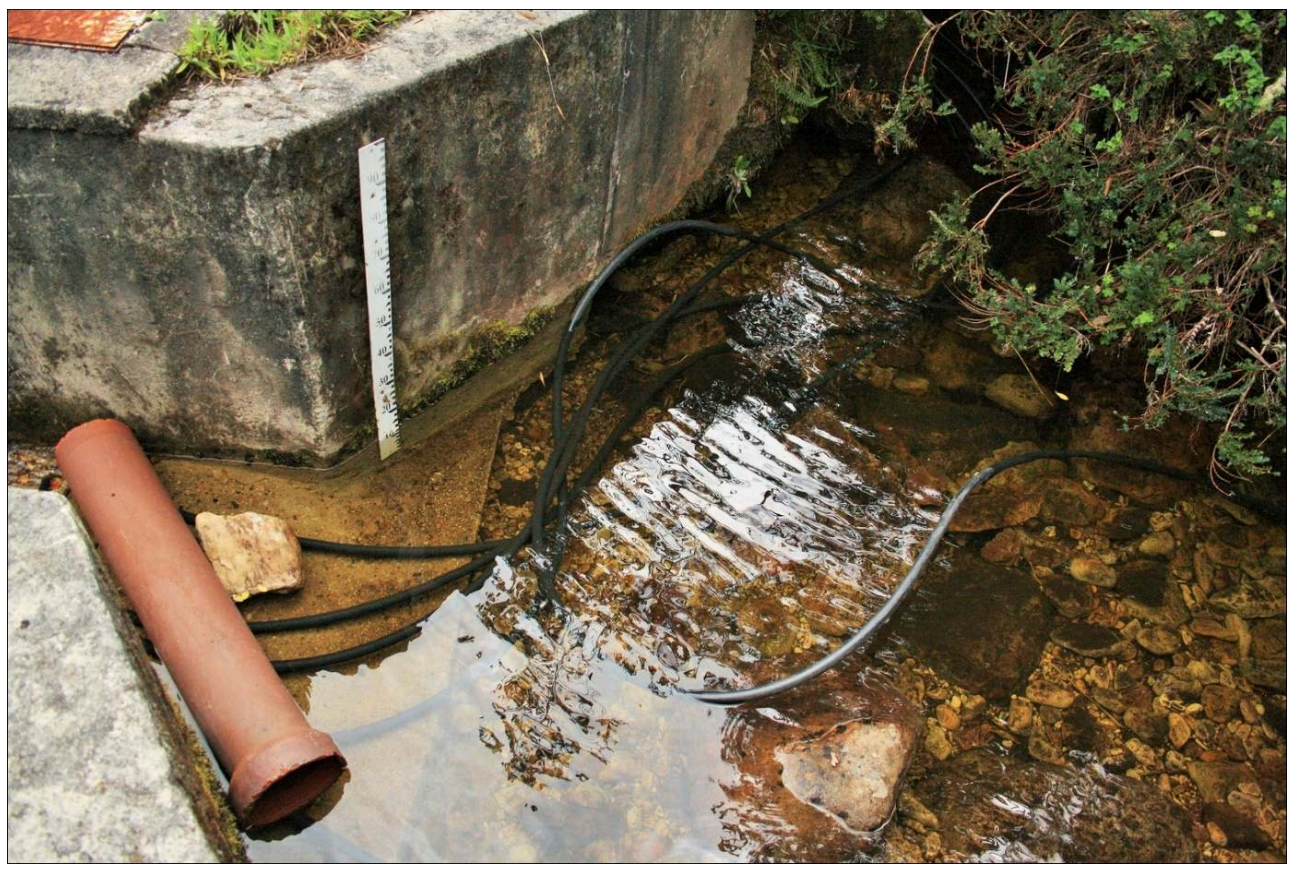

Illustration 9 - Les conditions de la flore autour de la source d'eau du réseau

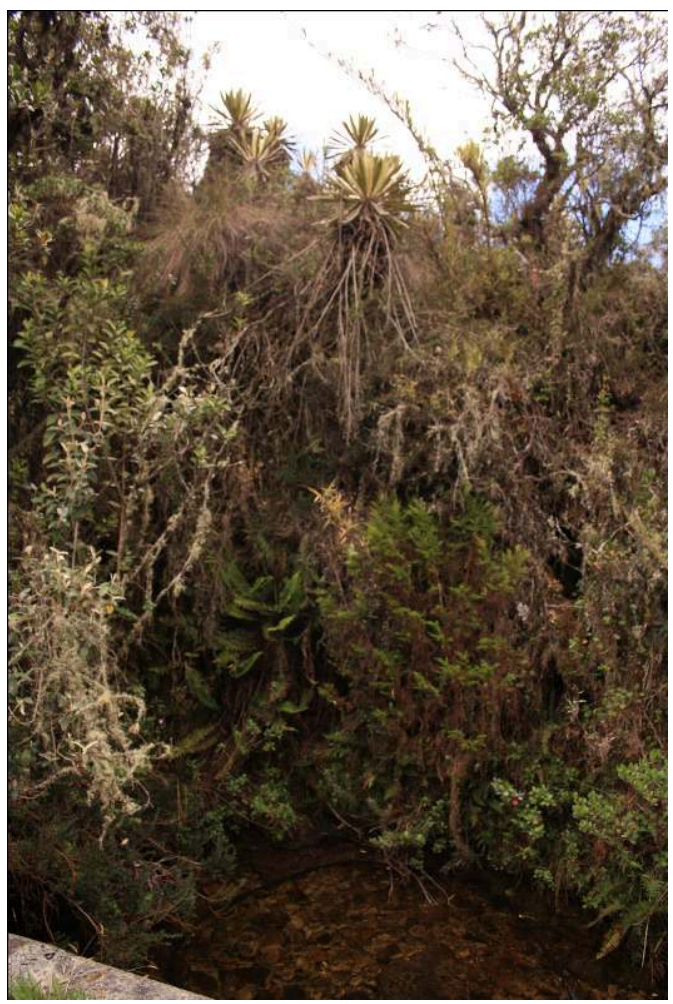

Par ailleurs, les membres du réseau ont choisi lors des assemblées générales annuelles un conseil d'administration (P3). Dans les assemblées un grand nombre de questions sont débattues comme, par exemple, les modifications des statuts (cf. extrait d'entretien avec le président du réseau) : 
«Les statuts ont été créés en fonction de ce qu'on voulait faire. Ils ont été créés avec l'aide d'un avocat et transmis à la Chambre de commerce. Après approbation de la communauté et signature on les retourne à la Chambre de commerce. Pour chaque modification faite aux statuts, on organise une Assemblée extraordinaire pour faire connaitre à la communauté les changements entrepris. Nous en avons fait une l'année dernière » (Président du réseau, Bogota, 2016). gestion réalisée par les gestionnaires du réseau, grâce aux rapports présentés. L'équipe qui gère le réseau est composée du président, de la trésorière et du plombier. Ces trois personnes sont employées et usagères du réseau. Les assemblées ont aussi pour but de permettre les échanges pour prendre des décisions qui vont garantir l'efficience, l'efficacité et la durabilité du réseau. Les deux extraits d'entretiens suivants expliquent le fonctionnement des assemblées (P4):

«Les Assemblées, avec tous les usagers, se font une fois par an et si besoin une deuxième peut avoir lieu, appelée " assemblée extraordinaire » (Usager du réseau, Bogota, 2016).

«Les assemblées générales sont faites tous les ans normalement. Elles sont organisées pour montrer tout ce qui a été fait dans l'année, par exemple pour montrer combien de mètres cubes d'eau ont été traités, ce que la communauté a consommé, à combien s'élèvent les contributions des usagers et combien d'argent on a reçu des subventions de l'État. On fait un bilan financier. On donne les rapports à la communauté qui a établi les statuts " (Président du réseau, Bogota, 2016).

Parmi les discussions lors des assemblées figure la validation de règles très précises pour l'utilisation de la ressource, notamment les quantités d'eau pouvant être consommées par usager et qui sont mesurées par des compteurs. Ceux qui ne les respectent pas font l'objet d'une plainte de la part du Conseil d'administration (P4). Puis, si les usagers continuent à enfreindre les règles, le plombier peut interrompre le service. Ce qui montre l'existence de sanctions graduées au sein du réseau (P5). Par contre, lorsque ce ne sont pas des usagers du service qui ne respectent pas les règles du réseau, notamment celle de ne pas cultiver en haut de la source d'eau pour éviter de polluer la ressource hydrique, le réseau ne peut pas imposer de sanctions. En effet, la législation nationale protège la propriété privée et donne aux propriétaires des terrains le droit d'utiliser ces terrains pour l'exploitation agricole. Ainsi, les sanctions imposées par le réseau pour garantir la durabilité de la gestion, sont limitées par ces lois. Cette situation est illustrée avec le cas de la culture de pomme de terre, comme le montre l'extrait d'entretien suivant :

«Ici il y a une grande production de pomme de terre. Il y a des personnes d'autres régions, comme Boyacá, qui viennent et qui achètent des grands terrains pour cultiver. Certains d'entre eux montent beaucoup dans les páramos et on a parlé avec eux pour qu'ils ne montent pas autant mais ils disent que ces terres leurs appartiennent, qu'ils payent les impôts, alors-ils ont le droit de cultiver de la pomme de terre là-bas » (Président du réseau, communication personnelle).

Le réseau compte aussi sur des mécanismes pour la résolution des conflits (P6). Lorsque des conflits émergent entre les usagers, la première instance auquel ils peuvent faire appel pour les résoudre est le plombier. Si le conflit n'a pas pu être résolu, il sera transmis au représentant légal. Puis, si besoin, le conflit fera l'objet d'une discussion lors de l'Assemblée générale avec prise de décision collective pour le régler. Cette dernière instance est le lieu où il est possible de résoudre les problèmes entre les usagers et les gestionnaires. En effet, ces situations nécessitent un échange entre les différentes parties et une prise de décision par l'Assemblée. Les deux extraits 
d'entretien suivants expliquent les causes des conflits dans le passé et comment des dispositifs mis en place aujourd'hui ont pu les résoudre :

« Maintenant il n'y a plus de problèmes entre les usagers et nous [les gestionnaires du réseau]. Avant, les problèmes existaient parce que tous les usagers n'avaient pas l'accès à l'eau de manière continue mais maintenant tous les usagers ont l'accès à l'eau de façon permanente » (Président du réseau, communication personnelle).

« Si jamais il y a un problème, on appelle le plombier et il vient pour le résoudre, il travaille tous les jours » (Usager du réseau communication personnelle).

Il persiste cependant quelques conflits beaucoup plus difficiles à résoudre : ce sont ceux qui existent entre le réseau et le gouvernement et qui sont liés au manque de reconnaissance des droits de l'organisation (P7). En effet, la législation nationale considère les réseaux alternatifs comme des entreprises, si bien que lorsqu'ils s'enregistrent auprès de la Chambre de commerce, les réseaux doivent se présenter comme tels (Superintendencia, 2018), ignorant leur caractère non lucratif. Cette situation se traduit par le manque d'appui technique qui représente un obstacle pour que les communautés aient accès à l'eau potable. Les demandes du réseau et les difficultés actuelles pour sa reconnaissance sont indiquées dans l'extrait d'entretien suivant :

« On œuvre pour que les institutions nous écoutent, on invite l'État à respecter le territoire et à reconnaître ses habitants comme des paysans. Mais même si les institutions nous sont favorables, si la loi dit une autre chose rien ne va changer... » (Usager, communication personnelle).

Enfin, le réseau Piedra Parada et Cerrito Blanco a créé des liens avec d'autres réseaux d'adduction d'eau non centralisés à partir de 2011 (P8). En effet, en 2011 RETACO s'est constitué pour faire face aux menaces de fermeture des réseaux liées aux critiques sur la qualité de l'eau qu'ils fournissaient. Ensuite, ils ont commencé à créer de liens avec d'autres réseaux au niveau national. Le lien créé avec RETACO est expliqué dans l'extrait d'entretien suivant:

«Nous avons commencé à nous associer avec RETACO parce qu'il y avait un politicien qui a planifié un débat pour fermer les réseaux communautaires et les vendre à une multinationale. Son argument de départ était de dire que nous fournissions de l'eau sale. Tous les réseaux se sont alors réunis pour aller contre le Conseil de Bogota parce que cet argument était faux et nous voulions nous battre pour défendre notre gestion. Le politicien avait même envoyé quelqu'un pour écouter ce qu'on disait et pour qu'il lui fasse un rapport. Nous avons expliqué que l'État ne faisait rien pour nous approvisionner en eau et qu'il ferait mieux de nous aider à avoir de l'eau de qualité plutôt que de critiquer notre gestion. Malgré ça, les lois nous déclarent en tant qu'entreprise privée alors que nous voulons être reconnus comme des gestionnaires d'un service public. Nous ne travaillons pas dans un but lucratif mais pour le bénéfice d'une communauté et non pour un intérêt personnel. L'argent que l'on gagne est investi dans le réseau lui-même et non pas pour un profit individuel » (Président du réseau, communication personnelle).

31 À partir de tout ce qui a été cité ci-dessus, il est possible d'affirmer qu'à des degrés variés la plupart des principes proposés par Ostrom (1990) sont respectés par le réseau Piedra Parada et Cerrito Blanco. C'est pourquoi ce réseau peut être considéré comme ayant les caractéristiques d'un réseau communautaire compatible avec l'environnement et durable. 


\section{Conclusions}

À travers le cas du réseau Piedra Parada et Cerrito Blanc, cet article a décrit le fonctionnement d'un système alternatif d'approvisionnement en eau dans les páramos de Bogota. Cette description a été réalisée à partir de la théorie des biens communs d'Elinor Ostrom (1990). Cette description me permet d'affirmer, qu'à des degrés variés, la plupart des principes proposés par Ostrom (1990) sont respectés par ce réseau. C'est pourquoi il peut être considéré comme ayant les caractéristiques d'un réseau communautaire, compatible avec l'environnement et durable. En effet, le réseau a des limites clairement définies en ce qui concerne le rayon d'approvisionnement (P1) ; il y a une cohérence entre les règles d'appropriation et de mise à disposition en conformité avec les conditions locales notamment au sujet des quantités approvisionnées aux usagers ( $\mathrm{P} 2)$; les assemblées générales permettent des règlements de conflits avec des choix collectifs (P3); il y a un suivi de la gestion et une supervision du respect des règles $(\mathrm{P} 4)$; si les règles ne sont pas respectées, des sanctions peuvent être imposées (P5) ; il y a des mécanismes de résolution des conflits (P6); le réseau est lié à d'autres institutions à plusieurs niveaux (P8). Cependant, à cause d'une législation qui ne reconnaît pas le travail à but non lucratif du réseau, le principe de reconnaissance minimal des droits de l'organisation n'est pas comblé (P7).

La présentation du réseau Piedra Parada et Cerrito Blanco a apporté des éléments relatifs à l'évolution de la quantité et de la qualité du service d'approvisionnement en eau des réseaux communautaires. Cependant, ce type de réseau, qui pourrait être au nombre de 12000 en Colombie (Cadavid, 2009), est toujours en dehors de la législation en vigueur et n'est pas encore reconnu par les institutions publiques comme un complément à la gestion publique. Cette analyse a également permis de constater que le réseau communautaire s'est constitué comme un espace de coordination d'actions concrètes pour la protection des páramos avec, par exemple, la construction de barrières qui limitent l'accès aux cours d'eau. De même, des actions collectives de restauration écologique sont menées comme le reboisement. Toutes ces actions démontrent que les réseaux communautaires d'eau ont un potentiel important dans la protection des páramos. C'est pourquoi, dans le cadre du processus de délimitation de ces systèmes socio-écologiques, des études approfondies doivent être menées dans d'autres régions de la Colombie. Ces études pourraient montrer s'il existe une présence historique des communautés dans les différents páramos, si les communautés se sont organisées et si elles ont un rôle de gestionnaires communautaires de l'eau et d'agents protecteurs de l'environnement. Les résultats de ces futures études pourraient être fondamentaux pour la délimitation des aires protégées en Colombie. Il serait également intéressant d'étudier de façon plus approfondie les savoirs et les pratiques des communautés locales (de tout le pays) pour pouvoir analyser, comparer et ainsi améliorer les connaissances actuelles en matière de protection et de restauration des pàramos. 


\section{BIBLIOGRAPHIE}

Acevedo-Tarazona Á., Correa Lugos A. D., 2019. Pensar el cambio socioambiental: Un acercamiento a las acciones colectivas por el páramo de Santurbán (Santander, Colombia). Revista Colombiana de Sociología [En ligne], vol. 42, n 1. URL: https://doi.org/10.15446/rcs.v42n1.73070

Alonso D. L., Pérez R., Okio C. K. Y. A. Castillo, E., 2020. Assessment of mining activity on arsenic contamination in surface water and sediments in southwestern area of Santurbán páramo, Colombia. Journal of Environmental Management [En ligne], vol. 264. URL: https://doi.org/10.1016/ j.jenvman.2020.110478

Álvarez-Fernández I., Freire J., Naya I., Fernández N., Sánchez-Carnero N., 2020. Failures in the design and implementation of management plans of Marine Protected Areas: An empirical analysis for the North-east Atlantic Ocean. Ocean \& Coastal Management [En ligne], vol. 192. URL: https://doi.org/10.1016/j.ocecoaman.2020.105178

Alvarez-Yela A. C., Alvarez-Silva M. C., Restrepo S., et al., 2017. Influence of agricultural activities in the structure and metabolic functionality of páramo soil samples in Colombia studied using a metagenomics analysis in dynamic state. Ecological Modelling [En ligne], vol. 351, p. 63-76. URL: https://doi.org/10.1016/j.ecolmodel.2017.02.010

Anthony B. P., Shestackova E., 2015. Do Global Indicators of Protected Area Management Effectiveness Make Sense? A Case Study from Siberia. Environmental Management, [En ligne], vol. 56, n 1, p. 176-192. URL: https://doi.org/10.1007/s00267-015-0495-z

Arrojo P., 2008. La nueva cultura del agua del siglo XXI. Zaragoza, Expoagua Zaragoza, 302 p.

Buitrago E., 2016. Limitaciones y delimitaciones de los páramos en una Colombia posacuerdo. In Ulloa A., Coronado S. (Edi) Extractivismos y posconflicto en Colombia: retos para la paz territorial. Bogotá, Universidad Nacional de Colombia, $434 \mathrm{p}$.

Cadavid N., 2009. Acueductos comunitarios: Patrimonio social y ambiental del Valle de Aburra. Avances en Recursos hidráulicos [En ligne], vol. 20, p. 57-64. URL: https://repositorio.unal.edu.co/ bitstream/handle/unal/8214/No.20-2009-5.pdf?sequence=1\&isAllowed=y

Cresso M., Clerici N., Sanchez A., Jaramillo F., 2020. Future Climate Change Renders Unsuitable Conditions for Páramo Ecosystems in Colombia. Sustainability [En ligne], vol. 12, n 20. URL: https://doi.org/10.3390/su12208373

Coutard O., 2010. Services urbains, la fin d'un dogme. In Pierre J., Tubiana L., Regards sur la Terre. Paris, Presses de Sciences Po, 340 p.

Cubillos-Pardo M. 2019. Nos páramos por el páramo: campesinos en oposición al extractivismo, San Bernardo, Sumapaz. Bogota, Pontificia Universidad Javeriana, 59 p.

Delgado-Serrano M., Ramos P., Lasso Zapata E., 2017. Using Ostrom's DPs as Fuzzy Sets to Analyse How Water Policies Challenge Community-Based Water Governance in Colombia. Water [En ligne], vol. 9, nº 7. URL: https://doi.org/10.3390/w9070535

Depraz S., 2008. Géographie des espaces naturels protégés. Paris, Armand Colin, 320 p.

Duarte-Ballesteros L., Urbina-Cardona J. N., Saboyá-Acosta L. P., 2021. Ensamblajes de anuros y heterogeneidad espacial en un ecosistema de páramo de Colombia. Caldasia [En ligne], vol. 43, n 1. URL: https://dx.doi.org/10.15446/caldasia.v43n1.84860. 
El Tiempo. October 28, 2010. Falla el 90 por ciento de los acueductos rurales. URL: https:// www.eltiempo.com/archivo/documento/CMS-8231450 (consulté le 26/07/2021).

Gil-Leguizamón P. A., Morales-Puentes M. E., Jácome, J., 2020. Estructura del bosque altoandino y páramo en el Macizo de Bijagual, Boyacá, Colombia. Revista de Biología Tropical [En ligne], vol. 68, n 3. URL: https://doi.org/10.15517/rbt.v68i3.34912

González-Martínez M. D., Huguet C., Pearse J., McIntyre N., Camacho L. A., 2019. Assessment of potential contamination of Páramo soil and downstream water supplies in a coal-mining region of Colombia. Applied Geochemistry [En ligne], vol. 108. URL: https://doi.org/10.1016/j.apgeochem. 2019.104382

Gutiérrez D. F., Fernández A., 2009. Proyecto Vidas Móviles: caracterización de las poblaciones en situación de desplazamiento y vulnerable receptora en la localidad de Ciudad Bolívar, Bogotá, D.C., Colombia. Universitas Medica [En ligne], vol. 50, $\mathrm{n}^{\circ}$ 1. URL: https://www.redalyc.org/ articulo.oa?id=231018725004

Gutiérrez-Díaz J. S., Ordoñez-Delgado N., Bolívar-Gamboa A., et al., 2020. Estimation of organic carbon in páramo ecosystem soils in Colombia. Ecosistemas [En ligne], vol. 29, $\mathrm{n}^{\circ}$ 1. URL: https:// doi.org/10.7818/ECOS.1855

Hopfe C., Ospina-Jara B., Scheibel T., Cabra-García J., 2020. Ocrepeira klamt sp. n. (Araneae: Araneidae), a novel spider species from an Andean páramo in Colombia. Plos one [En ligne], vol. 115, n 8. DOI: https://doi.org/10.1371/journal.pone.0237499

Llano-Arias, V. (2015). Community Knowledge Sharing and Co-Production of Water Services: Two Cases of Community Aqueduct Associations in Colombia. Water Alternatives [En ligne], vol. 8, $\mathrm{n}^{\circ} 2$. URL: https://www.water-alternatives.org/index.php/all-abs/282-a8-2-5/file

Londoño-Toro B., 2004. Bogotá: Una ciudad receptora de migrantes y desplazados con graves carencias en materia de recursos y de institucionalidad para garantizarles sus derechos. Estudios Socio-Jurídicos [En ligne], vol. 6, n 1. URL: http://www.scielo.org.co/scielo.php? script=sci_arttext\&pid=S0124-05792004000100011

Méndez-Polo O. L., 2019. Los intereses emergentes sobre la alta montaña y la vida campesina: Tensiones y contradicciones de la delimitación de páramos en Colombia. Cuadernos de Geografía: Revista Colombiana de Geografía [En ligne], vol. 28, n 2, p. 322-339. DOI: https://doi.org/10.15446/ rcdg.v28n2.70549

Montoya-Domínguez E., Rojas-Robles R., 2019. Normatividad del agua en Colombia :

¿democratización o privatización? Revista Luna Azul [En ligne], vol. 49, p. 126-145. DOI: https:// doi.org/10.17151/luaz.2019.49.7

Morales M., Otero J., Van der Hammen T. et al., 2007. Atlas de páramos de Colombia. Bogotá, Instituto de Investigación de Recursos Biológicos Alexander von Humboldt, 208 p.

Blanca, Nariño, Colombia. Perspectiva Geográfica [En ligne], vol. 22, n 2. DOI: https://doi.org/ 10.19053/01233769.7598

Olaya-Angarita, J. A., 2019. Composición y estructura de la transición bosque-páramo en el corredor Guantiva-La Rusia (Colombia). Revista de Biología Tropical [En ligne], vol. 67, nº 4. DOI: https://doi.org/10.15517/rbt.v67i4.31965

Ostrom E., 1990. Governing the commons: the evolution of institutions for collective action. Cambridge, Cambridge University Press, $280 \mathrm{p}$. 
Rivera D., Rodríguez C., 2011. Guía divulgativa de criterios para la delimitación de páramos de Colombia. Bogotá, Ministerio de Ambiente, Vivienda y Desarrollo Territorial, Instituto de Investigación de Recursos Biológicos Alexander von Humboldt, 68 p.

Secretaria Distrital del ambiente, 2021. Bogota es más campo que cemento (Ruralidad). URL: http:// ambientebogota.gov.co/de/ruralidad-sda (consulté le 01/04/2021).

Secretaría Distrital de Planeación, 2009. Conociendo la localidad de Ciudad Bolívar: Diagnóstico de los aspectos físicos, demográficos y socioeconómicos. URL: https://issuu.com/gobiernobogota/docs/ diagn_stico_engativ__2009 (consulté le 01/04/2021).

Soto-Vallejo I., Villarraga-Lozano Á. M., Cardona-Acevedo M., 2020. Gobernanza y servicios ambientales en la gestión de los acueductos comunitarios en tres municipios de Caldas, Colombia. Estudios Gerenciales [En ligne], vol. 36, n 155, p. 206-217. DOI: https://doi.org/10.18046/j.estger. 2020.155.3442

Superintendencia de servicios públicos domiciliarios (Superintendencia), 2018. Concepto 745 De 2018. URL: https://www.google.com/url?

$\mathrm{sa}=\mathrm{t} \& \mathrm{rct}=\mathrm{j} \& \mathrm{q}=\&$ esrc=s\&source=web\&cd=\&cad=rja\&uact=8\&ved=2ahUKEwiGwJb82YPyAhWADmMBHdIeCvoQFjAAegQIBRAD\&url=https\% (consulté le 27/07/2021).

Theobald D. M., Kennedy C., Chen B., et al., 2020. Earth transformed: Detailed mapping of global human modification from 1990 to 2017. Earth System Science Data, [En ligne], vol. 12, $\mathrm{n}^{\circ}$ 3, p. 1953-1972. DOI: https://doi.org/10.5194/essd-12-1953-2020

Therville C. 2013. Mise sous cloche ou intégration aux territoires? L'exemple des réserves naturelles de France. Brest, Université de Bretagne occidentale, 436 p.

Quiroz Dahik C., Crespo P., Stimm B. et al., 2021. Impacts of pine plantations on carbon stocks of páramo sites in southern Ecuador. Carbon Balance and Management, [En ligne], vol. 16, $\mathrm{n}^{\circ} 1$. DOI: https://doi.org/10.1186/s13021-021-00168-5

Villay-Quiñones H. A. 2018. Estrategias comunitarias para la conservación y uso sostenible de los de los servicios ecosistémicos del complejo de páramos Cruz Verde Sumapaz: caso de estudio provincia de Suacha, un aporte desde las prácticas culturales y los medios de vida. Bogotá, Universidad Piloto de Colombia, $196 \mathrm{p}$.

Yague-Páez E., 2012. Resistencia campesina al extractivismo en el páramo de Pisba: una propuesta desde la educación comunitaria para el fortalecimiento organizativo y la construcción de planes de vida comunitarios. Bogotá, Universidad Pedagogica Nacional, 158 p.

Zapata P. A., 2020. Estado actual de la delimitación de los páramos en Colombia, como mandato constitucional y su relación con la protección y conservación de estos ecosistemas estratégicos. Bogotá, Universidad de ciencias aplicadas y ambientales, $39 \mathrm{p}$.

\section{NOTES}

1. La ville de Bogota est subdivisée en 20 arrondissements et ceux-ci regroupent près de 2000 quartiers. Dans certains arrondissements, il y a des zones rurales et l'un des arrondissements, Sumapaz, est entièrement rural. 


\section{RÉSUMÉS}

La Colombie ne fait pas exception à la tendance mondiale de création et d'extension des aires protégées qui est illustrée par le processus de délimitation et de protection des páramos écosystème caractéristique des Andes septentrionales. L'inclusion des communautés locales dans cette délimitation occupe une place de plus en plus importante dans les débats politiques et scientifiques. Cet article vise à fournir des éléments pour comprendre la relation des communautés avec les páramos et leur rôle dans la protection de ces écosystèmes. Ce travail s'intéresse principalement aux systèmes alternatifs d'approvisionnement des services d'eau dans les páramos de Bogota, capitale du pays. À travers une étude de cas, ce travail montre que certains de ces systèmes sont communautaires, compatibles avec l'environnement et durables. La reconnaissance de ces systèmes en tant qu'agents protecteurs des paramos et fournisseurs de services d'eau apparaît comme une étape nécessaire pour la protection de l'environnement et la gestion des ressources naturelles du pays.

Colombia is no exception to the global trend of creation and extension of protected areas, which is illustrated by the process of delimitation and protection of the páramos -a characteristic ecosystem of the northern Andes-. The inclusion of local communities in this delimitation is becoming increasingly important in political and scientific debates. This article aims to provide elements to understand the relationship of communities with the páramos and their role in the protection of these ecosystems. This work focuses on alternative systems of water services provision in the páramos of Bogotá, the country's capital. Through a case study, this work shows that some of these systems are community-based, environmentally compatible and sustainable in the long term. The recognition of these systems as protectors of the paramos and providers of water services is a necessary step in protecting the environment and managing the country's natural resources.

\section{INDEX}

Mots-clés : gestion communautaire, eau, Páramo, Elinor Ostrom, aire protégée

Thèmes : Sur le Champ

Keywords : community-based natural resources management, water, Páramo, Elinor Ostrom, protected area

\section{AUTEUR}

\section{ANGELA OSORIO}

Angela Osorio, angela.osorio@live-cnrs.unistra.fr, est doctorante au Laboratoire Image Ville Environnement (LIVE UMR 7362), Université de Strasbourg. Elle a récemment écrit :

- Osorio A., 2017. La gestion comunitaria de « recursos naturales » en áreas protegidas. El caso de los acueductos comunitarios en los Páramos de Bogotá, Colombia. In Serrano-Sanz M., Gómez M. K. (ed). Políticas Públicas y Sociales: Globalización, desigualdad y nuevas insurgencias (Actes du colloque tenu à Université de Saragosse les 1 et 2juin 2017 à Saragosse, Espagne). Université de Saragosse, $367 \mathrm{p}$. 